\title{
Thin is required for cell death in the Drosophila abdominal muscles by targeting DIAP1
}

\author{
Kumar Vishal ${ }^{1}$, Simranjot Bawa', David Brooks ${ }^{1}$, Kenneth Bauman ${ }^{2}$ and Erika R. Geisbrecht ${ }^{1}$
}

\begin{abstract}
In holometabolous insects, developmentally controlled programmed cell death (PCD) is a conserved process that destroys a subset of larval tissues for the eventual creation of new adult structures. This process of histolysis is relatively well studied in salivary gland and midgut tissues, while knowledge concerning larval muscle destruction is limited. Here, we have examined the histolysis of a group of Drosophila larval abdominal muscles called the dorsal external oblique muscles (DEOMs). Previous studies have defined apoptosis as the primary mediator of DEOM breakdown, whose timing is controlled by ecdysone signaling. However, very little is known about other factors that contribute to DEOM destruction. In this paper, we examine the role of thin (tn), which encodes for the Drosophila homolog of mammalian TRIM32, in the regulation of DEOM histolysis. We find that loss of Tn blocks DEOM degradation independent of ecdysone signaling. Instead, th genetically functions in a pathway with the death-associated inhibitor of apoptosis (DIAP1), Dronc, and death-associated APAF1-related killer (Dark) to regulate apoptosis. Importantly, blocking Tn results in the absence of active Caspase-3 immunostaining, upregulation of DIAP1 protein levels, and inhibition of Dronc activation. DIAP1 and Dronc mRNA levels are not altered in th mutants, showing that Tn acts post-

transcriptionally on DIAP1 to regulate apoptosis. Herein, we also find that the RING domain of Tn is required for DEOM histolysis as loss of this domain results in higher DIAP1 levels. Together, our results suggest that the direct control of DIAP1 levels, likely through the E3 ubiquitin ligase activity of Tn, provides a mechanism to regulate caspase activity and to facilitate muscle cell death.
\end{abstract}

\section{Introduction}

Programmed cell death (PCD) governs the development and homeostasis of multicellular organisms by controlling the patterning of adult body structures and the removal of obsolete or damaged tissues ${ }^{1-4}$. Mechanisms by which cells die can be divided into three types based upon morphological criteria ${ }^{5}$. Type I PCD, or apoptosis, is characterized by the upregulation of caspases accompanied by DNA fragmentation, membrane blebbing, and

Correspondence: Erika R. Geisbrecht (geisbrechte@ksu.edu)

'Department of Biochemistry and Molecular Biophysics, Kansas State University, Manhattan, KS 66506, USA

2Department of Cell Biology and Biophysics, School of Biological Sciences, University of Missouri-Kansas, Kansas City, MO 64110, USA

Edited by E. Baehrecke cell rounding ${ }^{6}$. Autophagy is referred to as Type II PCD and is distinguished by the presence of doublemembraned autophagosomes ${ }^{7}$. Necrosis, the third type of PCD, is an inflammatory response characterized by cell swelling and rupture of the cell membrane ${ }^{8}$.

PCD is particularly evident during the metamorphosis of holometabolous insects, including Drosophila ${ }^{3}$, 9 . The first signs of apoptosis are observed in embryogenesis and persist into the pupal stages where many larval tissues are remodeled in response to pulses of the steroid hormone 20-hydroxy-ecdysone (ecdysone) ${ }^{9-11}$. A pulse of ecdysone in late third instar larvae (L3) promotes the larval to pupal transition, whereas a second pulse triggers $\mathrm{PCD}$ around $12 \mathrm{~h}$ after puparium formation (APF) ${ }^{12}$. After old tissues

\section{(c) The Author(s) 2018}

(c) Open Access This article is licensed under a Creative Commons Attribution 4.0 International License, which permits use, sharing, adaptation, distribution and reproduction c. in any medium or format, as long as you give appropriate credit to the original author(s) and the source, provide a link to the Creative Commons license, and indicate if changes were made. The images or other third party material in this article are included in the article's Creative Commons license, unless indicated otherwise in a credit line to the material. If material is not included in the article's Creative Commons license and your intended use is not permitted by statutory regulation or exceeds the permitted use, you will need to obtain permission directly from the copyright holder. To view a copy of this license, visit http://creativecommons.org/licenses/by/4.0/. 
are histolysed, newly formed tissues grow in the remaining 3.5 days before adult eclosion.

Most knowledge about tissue histolysis stems from the analysis of salivary gland or midgut tissue in pupal metamorphosis ${ }^{9}$. Salivary gland histolysis is regulated by the late ecdysone pulse ${ }^{13-15}$, whereby ecdysone binding to its receptors promotes the expression of early genes, including the transcription factors Broad complex $(\mathrm{Br}-\mathrm{C})$, $E 74 A$, and $E 93^{16-19}$. Subsequent activation of cell death genes, including hid, reaper, Dronc, Drice, and the autophagy-related gene 1 (Atg1), result in the elimination of this tissue $\mathrm{e}^{9,14,15,17,20,21}$. While salivary gland histolysis is mediated by both apoptosis and autophagy ${ }^{22}$, midgut histolysis is triggered by the early ecdysone pulse and is primarily regulated by autophagy ${ }^{23}$. Less is understood about muscle remodeling during pupation, including the signaling pathways that control whether muscle cells are fated to live or die.

Drosophila makes two sets of muscles during its life cycle, one in embryogenesis for larval movement and the other during pupation for adult life. During metamorphosis, most of the larval muscles are histolyzed and this pupal remodeling assures muscles are functional for adult-specific functions like flight and mating ${ }^{24,25}$. Two sets of muscles that undergo remodeling during the pupal transition are the dorsal internal oblique muscles (DIOMs) and the dorsal external oblique muscles $(\mathrm{DEOMs})^{26,27}$. Both of these muscle groups are present in abdominal segments A1 to A5. The muscles closest to the midline are designated as DIOM1 or DEOM1, whereas more lateral muscles are classified as DIOM2 or DEOM2 (Fig. 1a, b). DIOMs fail to undergo histolysis and persist until adult stages, whereas the DEOMs are removed by $\mathrm{PCD}^{26,28}$. DEOM1 histolysis is initiated by $8 \mathrm{~h}$ APF and the muscles are lost by $12 \mathrm{~h}$ APF. DEOM2 histolysis is delayed and is typically completed by $24 \mathrm{~h} \mathrm{APF}^{28-30}$.

Destruction of the DEOMs is mediated solely by apoptosis ${ }^{28}$. Transmission electron microscopy studies show myonuclei containing condensed chromatin in dying DEOMs and ectopic expression of the antiapoptotic protein p35 in DEOMs is sufficient to prevent muscle breakdown. In contrast, muscle-specific reduction of the autophagy gene Atg1 has no effect ${ }^{28}$. Like salivary gland and midgut histolysis, ecdysone signaling mediates DEOM breakdown, which is evidenced by a reduction in caspase staining and the absence of muscle histolysis upon loss of the ecdysone receptor ${ }^{28}$.

The core machinery required for apoptosis is conserved among flies, worms, and mammals ${ }^{2,} 31$. The caspase family of proteins are the principal mediators of cell death and are present in most cells in an inactive form ${ }^{32,33}$. Under normal conditions, caspase activity is blocked by the inhibitor of apoptosis (IAP) family of proteins to prevent cell death $^{34,35}$. In Drosophila, Dronc (Caspase-9) acts as the principal initiator caspase and is inhibited by DIAP $1^{36-38}$. Upon receiving a cell death stimulus, the IAP antagonists Reaper, Hid, and Grim (RGH) promote the degradation of DIAP1, resulting in Dronc release ${ }^{39-43}$. A Dronc-Dark complex form the apoptosome to activate the executioner caspases Drice and Dcp-1, which cleave cellular substrates to promote cell death ${ }^{44,45}$.

Herein, we have discovered that $\mathrm{Tn}$ acts in a pathway with DIAP1 and Dronc to regulate abdominal muscle breakdown. $t n$, also called another B-box affiliate $(a b b a)$, is homologous to mammalian TRIM32 and is characterized by an N-terminal RING domain followed by six NHL repeats at the $\mathrm{C}$ terminus ${ }^{46,}{ }^{47}$. The RING domain provides E3 ubiquitin ligase activity, whereas the NHL repeats are predicted to facilitate protein-protein interactions. Pleiotropic roles exist for the ubiquitously expressed TRIM32 protein in regulating muscle physiology, muscle regeneration, and tumor suppression ${ }^{46,47}$. Our results here demonstrate a role for Tn in controlling the fate of muscle cells, acting as a switch to control whether muscle cells live or die.

\section{Results}

Tn is required for muscle histolysis and is not an ecdysone target

We previously showed that $\mathrm{Tn}$ is required for myofibril stability and costamere integrity in Drosophila larval muscles $^{48}$. However, the late pupal lethality of $t n$ mutants suggested that Tn may be required during pupal metamorphosis. Indeed, targeting tn RNAi in the musculature using the Gal4/UAS system produced defects in abdominal muscle histolysis.

At $0 \mathrm{~h} \mathrm{APF}$, all DEOMs were present in both control (mef2-Gal4/+) and experimental (mef2>tn RNAi) genotypes (Fig. 1c-e). Around $8 \mathrm{~h} \mathrm{APF}$, smaller, rounded muscles indicated the onset of DEOM1 histolysis in controls (Fig. 1f, h), while DEOM1 was still fully intact upon a decrease in Tn (Fig. 1g, h). By 12 h APF, DEOM1 was absent in nearly all mef2-Gal4/+ individuals (Fig. 1i, k), while loss of DEOM1 was observed only in approximately $28 \%$ of th $R N A i$ abdominal segments (Fig. 1j, k). Complete histolysis of DEOM1 (and DEOM2) were apparent in controls at $24 \mathrm{~h}$ APF (Fig. 1l, n). In contrast, a partial block in degradation was observed in DEOMs with disrupted Tn function at $24 \mathrm{~h} \mathrm{APF} \mathrm{(Fig.} \mathrm{1m,}$ n). This block in DEOM histolysis was verified in $t n^{\Delta A}-/$ - mutants $(t n-/-)$ and an additional tn RNAi line (Fig. S1). Thus, we conclude that the abrogation of Tn results in impaired muscle histolysis.

As ecdysone signaling directs tissue histolysis during metamorphosis ${ }^{9}$, we sought to examine if $\mathrm{Tn}$ is an ecdysone target. tn $m R N A$ levels were measured in pupae with blocked ecdysone signaling prior to ( $0 \mathrm{~h} \mathrm{APF),} \mathrm{during}$ (12 h APF), or after (24h APF) completion of histolysis 


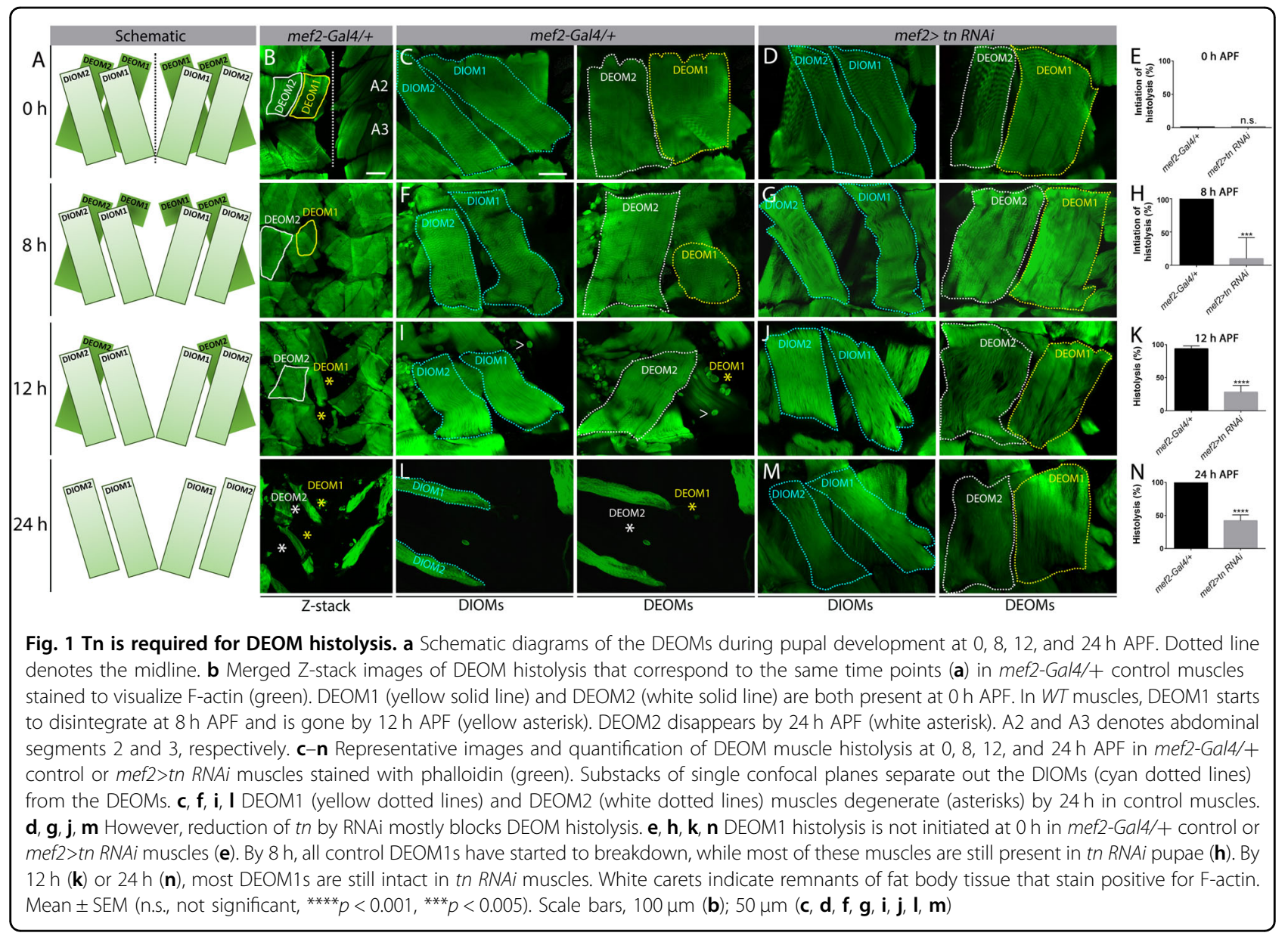

using quantitative PCR (qPCR). There was no significant difference in $t h$ transcript levels between mef2-Gal4/+ or $m e f 2>D N-E c R B 1$ samples at any stage of development (Fig. 2a). The reduction in $t n$ transcript levels in $m e f 2>t n$ $R N A i$ muscles is consistent with previous results ${ }^{49}$, further demonstrating the specificity of inducing tn RNAi in pupal muscles and the sensitivity of our qPCR approach. Our data demonstrate that ecdysone does not globally regulate $t h$ expression during metamorphosis.

To test if Tn plays a broader role in general tissue histolysis, we examined whether Tn regulates midgut or salivary gland breakdown. The midgut undergoes a drastic reduction in size between the late L3 and early pupal stages, whereas salivary gland histolysis takes place between $12 \mathrm{~h} \mathrm{APF}$ and $16 \mathrm{~h} \mathrm{APF}^{50}$. WT and $t n-/-$ midguts appeared similar at $4 \mathrm{~h} \mathrm{APF} \mathrm{(Fig.} \mathrm{2b-e)} \mathrm{and} \mathrm{there} \mathrm{was}$ no obvious delay or impairment in salivary gland histolysis in th mutants compared to $W T$ at $16 \mathrm{~h}$ APF (Fig. 2f-i). Therefore, unlike abdominal muscle, Tn does not play a broader role in general tissue breakdown during metamorphosis.

\section{Loss of Tn affects DIAP1 protein levels and Caspase-3 activity}

Zirin et al ${ }^{28}$ previously demonstrated that disintegration of the DEOMs relies on apoptosis. To understand if Tn facilitates DEOM cell death, we assayed protein levels of the initiator caspase Dronc since antibodies against cleaved-Caspase-3 are a read-out of Dronc activity ${ }^{51}$. Elevated Caspase-3 was present in mef2-Gal4/+ muscles undergoing histolysis at $12 \mathrm{~h}$ APF (Fig. 3a, c). In contrast, there was an overall reduction in puncta corresponding to active Caspase- 3 in muscles with disrupted Tn function (Fig. 3b, c). Next we examined DIAP1 protein levels. At $12 \mathrm{~h}$ APF, DIAP1 was present in DEOM controls at a basal level (Fig. 3d, f), whereas significantly higher DIAP1 levels were observed in tn RNAi DEOMs (Fig. 3e, f). This observed reduction in Dronc activity and the elevation of DIAP1 in th mutants was not due to altered mRNA expression of DIAP1, Dronc, Drice, or Dark (Fig. S2). These data suggest that $\mathrm{Tn}$ post-transcriptionally regulates some of the cell death genes to direct muscle histolysis. 

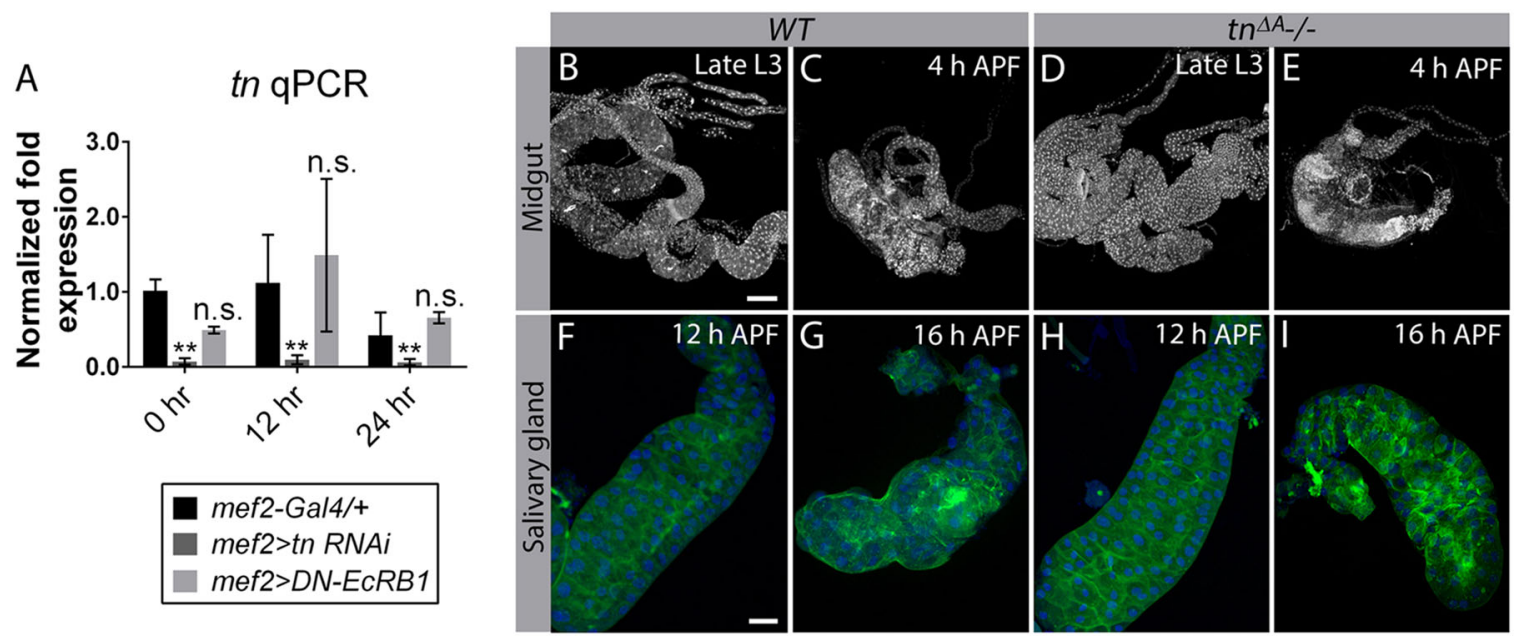

Fig. $2 \mathrm{Tn}$ is not an ecdysone target and is not required for general tissue histolysis. a tn mRNA levels measured by qPCR are not significantly different from controls (mef2-Gal4/+) when a dominant-negative version of isoform EcRB1 is expressed in muscle tissue during pupal morphogenesis. Note that $t n$ transcript levels are decreased as expected upon muscle-specific tn RNAi knockdown. $N=3$ biological replicates and 3 technical replicates for each genotype. $\mathbf{b}$-i Z-stack confocal images of midguts $(\mathbf{b}-\mathbf{e})$ or salivary glands $(\mathbf{f}-\mathbf{i})$. Midgut histolysis proceeds normally by 4 h APF in WT (b, $\mathbf{c})$ or tn mutants (d, e) as assayed by DAPI staining. PCD still occurs in salivary glands (F-actin, green and DAPI, blue) by $16 \mathrm{~h}$ APF upon loss of Tn (h, i) compared to WT controls $(\mathbf{f}, \mathbf{g})$. Mean \pm SEM (n.S., not significant, $\left.{ }^{* *} p<0.01\right)$. Scale bars, $200 \mu m(\mathbf{b}-\mathbf{e})$ and $100 \mu m(\mathbf{f}-\mathbf{i})$

Since overexpression (OE) of the cell death inhibitor p35 blocks DEOM breakdown ${ }^{28}$, we next examined p35 to assess if Tn generally influences protein levels during histolysis or specifically functions in DIAP1-mediated apoptosis. The baculovirus p35 protein is not endogenously expressed in Drosophila, but inhibits effector caspases $^{52}$. Thus, we used the Gal4/UAS system to target p35 in muscle alone (mef2>p35) or in a tn RNAi background (mef2>tn $R N A i+p 35 O E$ ). There was no significant difference in p35 levels in either genotype at $12 \mathrm{~h}$ APF (Fig. 3g-i). Moreover, there was no increase in the fluorescence intensity of DIAP1 upon p35 OE in the DEOMs (Fig. S3), proving that the observed accumulation of DIAP1 protein in $t n-/-$ is not a secondary consequence of a block in muscle histolysis. Collectively, these findings show that $\mathrm{Tn}$ regulates DEOM cell death by influencing DIAP1 protein levels and Dronc activity.

\section{tn functions in a pathway with cell death genes}

In the presence of cell death signals, the pro-apoptotic RGH proteins block DIAP1 inhibition, thereby enabling Dronc activation ${ }^{2,35}$. Stable Dronc protein is activated by Dark and this Dronc-Dark apoptosome complex promotes the activity of effector caspases such as Drice for the execution of cell death (Fig. 4a) ${ }^{44,45}$. Since our results show that tn RNAi blocks DEOM histolysis by altering DIAP1 and Caspase-3, we next sought to genetically manipulate cell death pathway components in a $t n R N A i$ background to determine if $\mathrm{Tn}$ directly functions in apoptosis. Cell death was first blocked by expressing the cell death inhibitors DIAP1 (mef2>DIAP1 OE) or p35 (mef2>p35 OE). By $12 \mathrm{~h}$ APF, all DEOM1s underwent histolysis in mef2-Gal4/+ controls (Figs. 1i, k and 4b). Muscle-targeted DIAP1 OE inhibited degradation in approximately $50 \%$ of DEOM1s (Fig. 4c, h). In a tn RNAi background, DIAP1 OE did not enhance the extent of DEOM1 histolysis (Fig. 4f, h), suggesting that $t n$ and DIAP1 may be acting in concert to regulate muscle degradation. In contrast, exogenous expression of $\mathrm{p} 35$ was more effective in the prevention of DEOM1 degeneration alone (Fig. 4d, i) and in combination with tn $R N A i$ (Fig. 4g, i), presumably due to the ability of p35 to block three different effector caspases (Drice, Dcp-1, and Decay $)^{3}$. The addition of exogenous UAS elements (UASGFP OE or UAS-GFP RNAi) in the same tn RNAi background did not alter DEOM1 histolysis, indicating that sufficient Gal4 protein is present to drive all UAS-based constructs in the presence of UAS-tn RNAi (Fig. S1).

Next, we utilized muscle-specific RNAi to silence Dronc (mef2>Dronc RNAi) or Dark (mef2>Dark RNAi) to mimic a reduction in apoptotic signaling at 12 APF. In contrast to blocking apoptosis through OE of DIAP1 or p35, RNAi knockdown of Dark (Fig. 5b, d) or Dronc (Fig. 5f, h) alone was less effective at blocking DEOM1 histolysis. qPCR analysis revealed that Dark (Fig. 5a) and Dronc (Fig. 5e) transcript levels were decreased by $\sim 60 \%$ under control of the ubiquitous daughterless (da)-Gal4 promoter, likely accounting for the weaker block in histolysis than DIAP1 OE. The extent of muscle histolysis was not enhanced in a $t n R N A i$ background upon a further reduction in Dark (Fig. 5c, d) or Dronc (Fig. 5g, h), further supporting the model that Tn acts with cell death genes to regulate DEOM histolysis. 

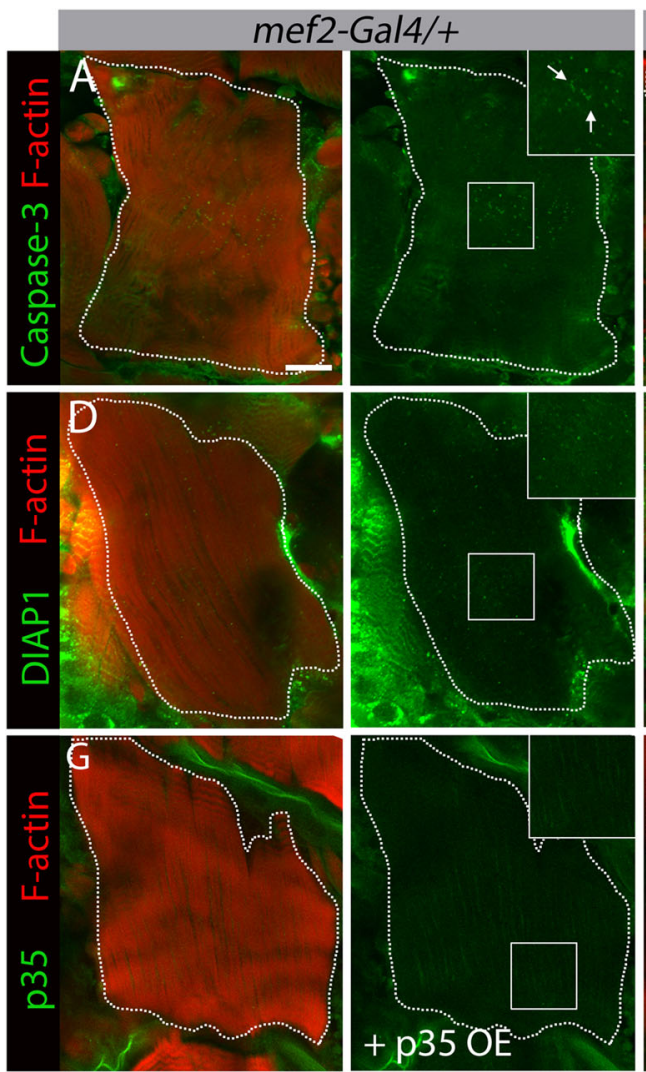
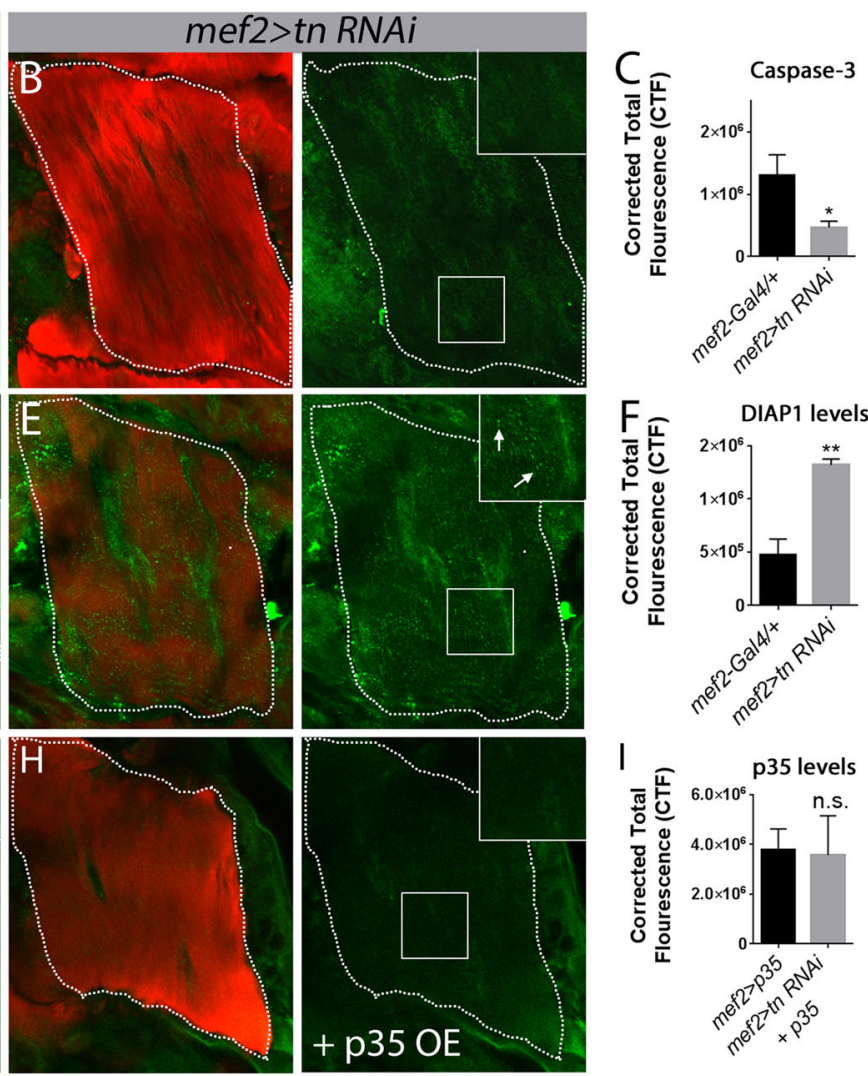

Fig. 3 Tn affects DIAP1 and Caspase-3 activity during DEOM histolysis at $12 \mathbf{~ h ~ A P F . ~ a - c ~ I n ~ v i v o ~ l a b e l i n g ~ o f ~ C a s p a s e - 3 ~ ( g r e e n ) ~ i n ~ m e f 2 - G a l 4 / + ~}$ control or mef2>tn RNAi DEOMs co-labeled with phalloidin (red). a Puncta corresponding to Caspase-3 are present in degenerating DEOM controls (white arrows in inset). b No Caspase-3(+) puncta are present in tn RNAi muscles. c Quantification of the relative fluorescence intensities of Caspase-3 in DEOMs reflects the general decrease observed upon a reduction in Tn. $\mathbf{d}-\mathbf{f}$ Immunostaining for DIAP1 (green) and F-actin (red) is higher upon disruption of Tn function (e, white arrows in inset) than in mef2-Gal4/+ control muscles (d). $\mathbf{f}$ Bar graph showing that the relative DIAP1 fluorescence levels are increased upon reduced Tn function. g-i Overall p35 (green) levels in DEOMs labeled with F-actin (red) are not changed upon a reduction in Tn. mef2>p35 appears similar when co-expressed in a WT (g) or tn RNAi (h) background. Quantification of fluorescence intensity reveals no significant difference between WT or experimental DEOMs (i). Mean \pm SEM (n.s., not significant, ${ }^{* *} p<0.01,{ }^{*} p<0.05$ ). Scale bar, $50 \mu \mathrm{m}$

Due to this partial block of Dark or Dronc caspase activity using RNAi, we next examined loss-of-function alleles. Both homozygous Dark $^{L 46}$ and Dronc $^{I 29}$ individuals are partially pupal lethal ${ }^{53,54}$ but survive through muscle remodeling. At 12 APF, DEOM1 histolysis was blocked in both Dark (Fig. 5k, m) and Dronc (Fig. 5l, m) mutants, although to a lesser extent than $t n-/-$, where muscle degradation was completely abolished (Fig. 5j, m). At $24 \mathrm{~h}$ APF when DEOM1 and DEOM2 were normally absent in $W T$ pupae (Fig. $5 n, r)$, the majority of DEOM2s failed to undergo histolysis upon loss of Tn (Fig. 5o, r), Dark (Fig. 5p, r), or Dronc (Fig. 5q, r). Together, these results show that $T n$ mediates muscle breakdown by acting through the DIAP1-Dronc pathway.

\section{The RING domain of $\mathrm{Tn}$ is required for DEOM histolysis}

Drosophila Tn contains a conserved N-terminal RING domain and six NHL repeats in the $\mathrm{C}$ terminus (Fig. 6a). The B-Box and coiled-coiled (CC) regions are poorly conserved $^{48}$. The requirement for the RING and NHL regions was investigated using genetic rescue experiments. We examined the effects of expressing full-length Tn (Tn FL) or versions of Tn lacking the RING ( $t n \Delta R I N G)$ or NHL domains $(t n \Delta N H L)$ in DEOMs subjected to th RNAi at $24 \mathrm{~h}$ APF. Transgene expression was confirmed by Tn immunostaining in the DEOMs and qPCR for $t n$ transcript quantitation (Fig. S4). As expected, restoration of DEOM histolysis was observed upon the introduction of Tn FL in a tn RNAi background (Fig. 6d, g) compared to th RNAi alone (Fig. 6c, g). DEOM1 was still intact at 24h APF upon loss of the RING domain in Tn (Fig. 6e, g), while the presence of the RING domain $(m e f>t n \quad R N A i+t n \triangle N H L)$ restored DEOM histolysis (Fig. 6f, g). These results indicate that the RING domain of $\mathrm{Tn}$ is required to prevent muscle breakdown.

The RING domain in E3 ubiquitin ligases such as Tn is required to transfer ubiquitin moieties from an E2 enzyme to a target substrate for proteasomal degradation ${ }^{55}$. Thus, 


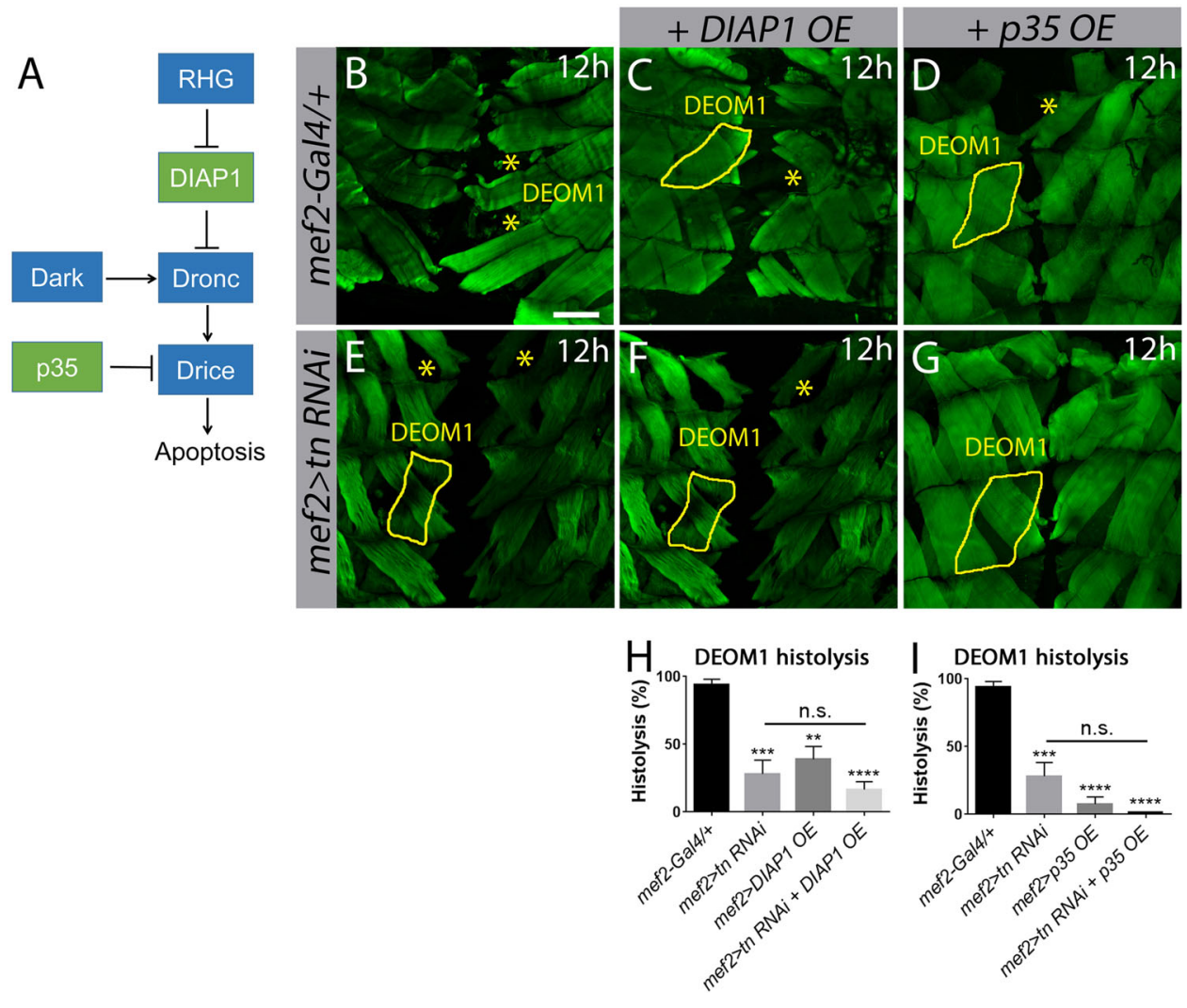

Fig. 4 tn genetically interacts with DIAP1 during DEOM histolysis. a Schematic of Drosophila core cell death machinery. b-g Merged Z-stack confocal images of the abdominal muscles stained with phalloidin (green) at $12 \mathrm{~h}$ APF. DEOM1 is outlined with a solid yellow line and histolysed DEOM1s are marked by yellow asterisks. b Histolysis proceeds normally in mef2-Gal4/+ muscles. Overexpression of DIAPI (c) or p35 (d) in muscles partially blocks DEOM1 histolysis. $\mathbf{e}-\mathbf{g}$ There is no significant difference in DEOM1 histolysis upon RNAi knockdown of th alone (e), or with overexpression of DIAP1 (f) or p35 (g) in a tn RNAi background. $\mathbf{h}$ A bar graph showing similar levels of muscle histolysis in mef2>tn RNAi+DIAP1 OE compared to mef2>tn RNAi muscles. i Quantification showing no significant difference in DEOM1 breakdown between mef2>tn RNAi and mef2>tn RNAi+p35 OE pupae. Mean \pm SEM (n.s., not significant, $\left.{ }^{* * * *} p<0.001,{ }^{* * *} p<0.005,{ }^{* *} p<0.01\right)$. Scale bar, $100 \mu m(\mathbf{b}-\mathbf{g})$

to test if the RING domain of Tn may regulate DIAP1 protein levels through a similar mechanism, we examined DIAP1 immunostaining in DEOMs at $12 \mathrm{~h}$ APF again using truncated Tn constructs. Consistent with previous results in Fig. 3e, DIAP1 protein was elevated upon a reduction in Tn (Fig. 6i, m). Expression of Tn FL (Fig. 6j, $\mathrm{m}$ ) or Tn lacking the NHL region (Fig. 6l, m) reduced DIAP1 levels similar to control muscles (Fig. 6h, m). In contrast, significantly higher DIAP1 was present in DEOMs expressing Tn $\triangle$ RING in a tn RNAi background (Fig. 6k, m). These results substantiate the importance of the RING domain in DEOM histolysis, specifically suggesting that DIAP1 is a substrate for Tn-mediated E3 activity.

\section{Tn acts via DIAP1 to regulate Dronc activity}

To further verify that loss of Tn alters DIAP1 levels, we performed western blots to quantify DIAP1 protein in WT or $t n-/-$ pupae. Before the initiation of DEOM destruction $(0 \mathrm{~h} \mathrm{APF})$, there was no significant difference in DIAP1 levels between WT or th mutants (Fig. 7a, b). However, at $8 \mathrm{~h} \mathrm{APF,} \mathrm{DIAP1} \mathrm{levels} \mathrm{were} \mathrm{approximately} \mathrm{2-}$ fold higher upon loss of Tn. Since a primary role for DIAP1 is to bind and inhibit Dronc activity, we next examined Dronc processing. During apoptosis, FL Dronc is cleaved to produce Pr1 and Pr2 forms ${ }^{5-58}$. DIAP1 physically interacts with the FL and Pr1 forms of Dronc, thus preventing further cleavage to the active Pr2 protein. In $W T$ pupae at $24 \mathrm{~h} \mathrm{APF}$, both the FL and processed Pr1 and Pr2 Dronc proteins were present (Fig. 7c). However, only the active form of Dronc was present from $0 \mathrm{~h}$ to $12 \mathrm{~h}$ $\mathrm{APF}$, consistent with the normal timing of DEOM muscle histolysis. Interestingly, loss of Tn resulted in solely the Pr1 form (Fig. 7c). These data support a model whereby the upregulation of DIAP1 protein upon Tn deficiency prevents full Dronc activation, thus preventing apoptosis.

\section{Discussion}

PCD is required for the destruction of certain larval tissues during metamorphosis ${ }^{4,} 9$, 59. Zirin et al. ${ }^{28}$ 


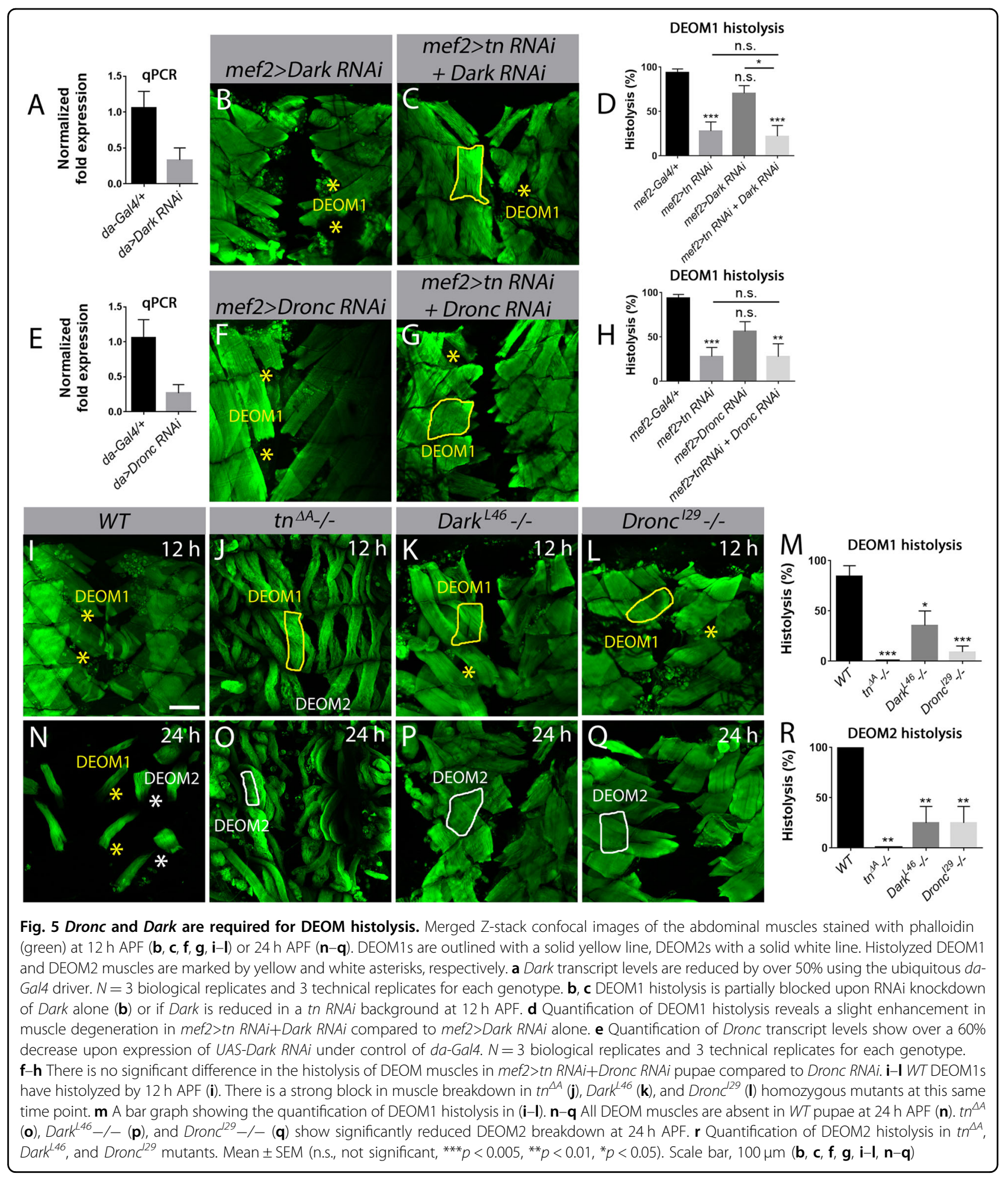

established that histolysis of the abdominal muscles is regulated by apoptosis, while blocking autophagy does not affect muscle breakdown ${ }^{28}$. In addition to the ecdysone receptor, only a handful of nuclear proteins are known to function in DEOM histolysis. Loss of East results in a partial block in DEOM degeneration, whereas premature muscle destruction is observed in muscles that lack Chromator ${ }^{27}$. Moreover, the two nuclear receptors, FTZF1 and HR39, antagonistically function to regulate the timing of DEOM histolysis ${ }^{28}$. Here we have further 


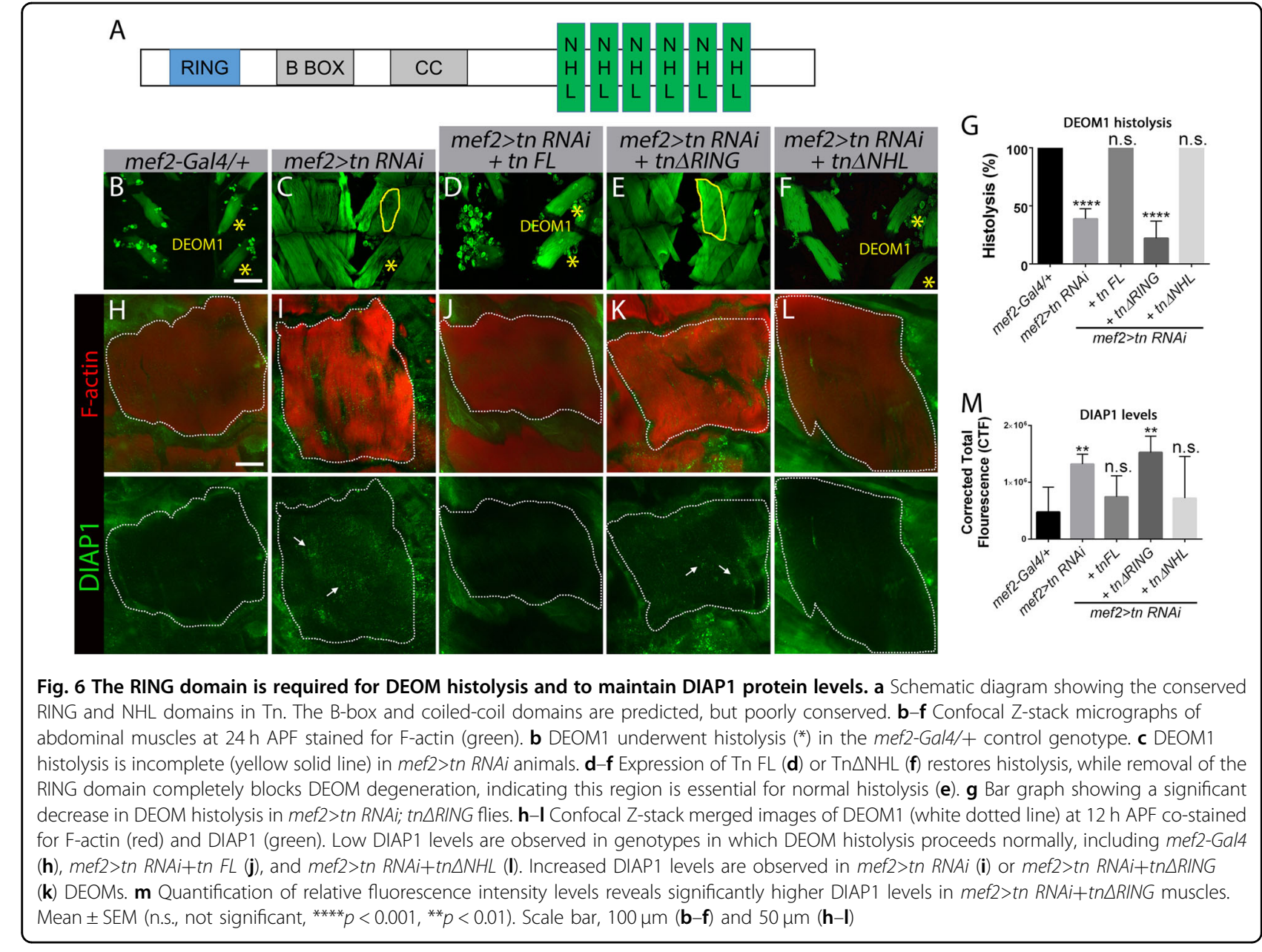

\begin{tabular}{|l|l|l|l|l} 
B BOX & & CC \\
\hline
\end{tabular}
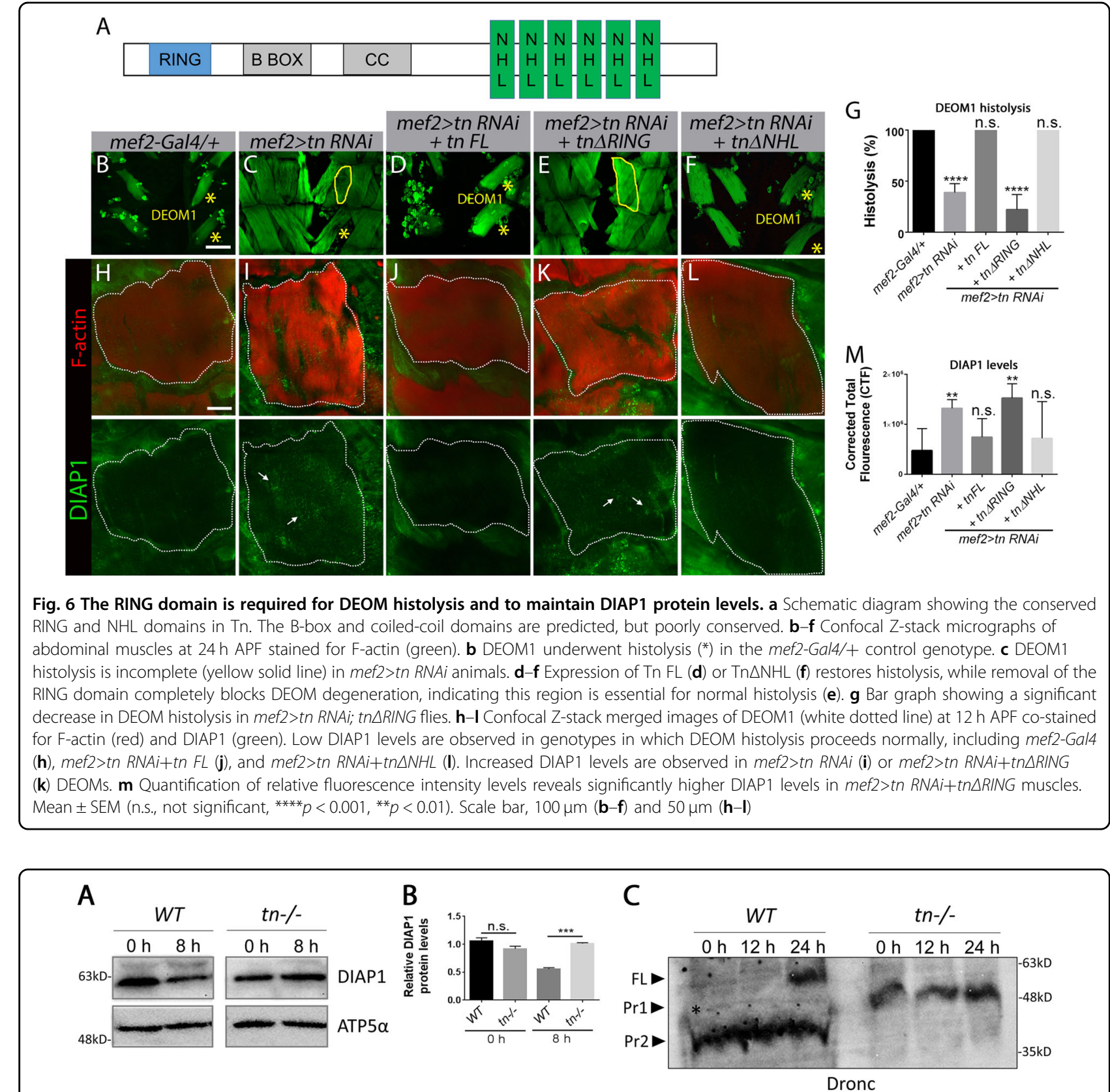

Fig. 7 Loss of Tn affects DIAPI protein levels and Dronc activation. a Total protein extracted from pupae at 0 or $8 \mathrm{~h}$ APF was subjected to western blotting to assess DIAP1 or ATP5a protein levels. DIAP1 levels at $8 \mathrm{~h}$ APF are increased in tn mutants. $\mathbf{b}$ Densitometry quantification of the relative DIAP1 protein levels using ATP5a as a loading control reveals a significant increase in DIAP1 protein at $8 \mathrm{~h}$ upon loss of Tn ( $n=3)$. $\mathbf{c}$ Dronc processing in $W T$ or $t n-/-$ pupae. Dronc FL $(\sim 55 \mathrm{kDa})$ is present at $24 \mathrm{~h}$ APF in $W T$ samples. The cleaved Pr 1 form $(\sim 40 \mathrm{kDa})$ is weakly observed during WT metamorphosis (black asterisk), while the active Pr2 cleavage product of Dronc ( $37 \mathrm{kDa})$ predominates from 0 to $24 \mathrm{~h}$ APF. Only the Pr1 form is present upon loss of Tn throughout DEOM histolysis. Mean \pm SEM (n.S., not significant, ${ }^{* *} p<0.005$ )

identified Tn as a novel protein in pupal muscle remodeling. However, loss of Tn does not affect salivary gland and midgut histolysis, highlighting an exclusive muscle role for Tn during Drosophila metamorphosis.

Our genetic assays demonstrate that $t n$ functions with core components of the cell death machinery to regulate
DEOM destruction. It was surprising that inhibition of apoptotic activity in Dark or Dronc mutants was not sufficient to completely block histolysis by $24 \mathrm{~h}$ APF. One explanation is the existence of additional cell death mechanisms other than apoptosis. While histolysing DEOMs contained autophagic vesicles, a reduction in 

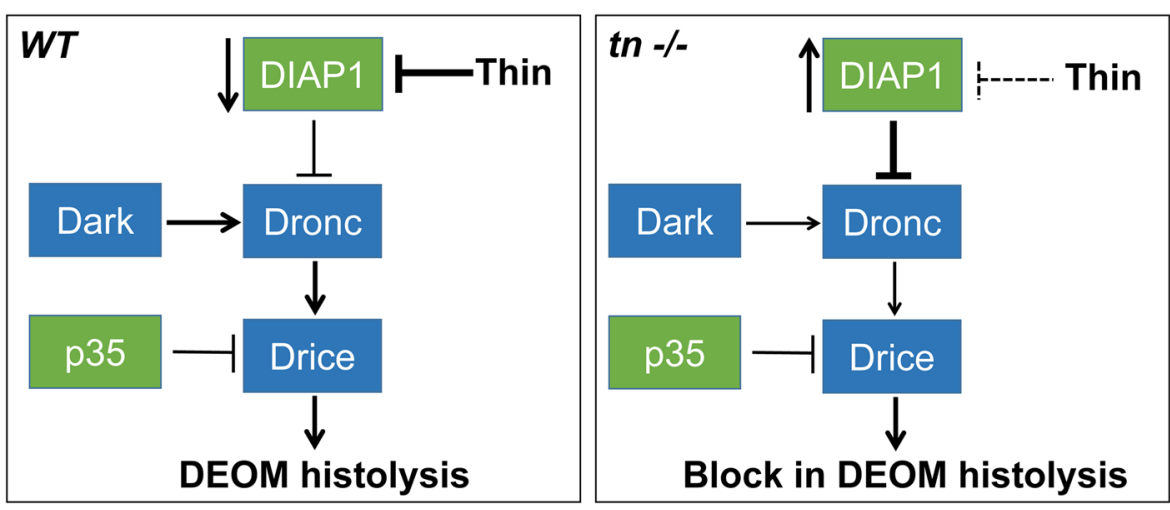

Fig. 8 Model for Tn-mediated regulation of DEOM histolysis. a In WT DEOMs, Tn targets DIAPI for degradation. Downregulation of DIAP1 results in DEOM histolysis via caspase-dependent apoptosis. $\mathbf{b}$ In the absence of Tn, DIAPI levels increase and block caspase activity. Lack of caspase activation blocks cell death and preserves DEOMs

autophagy components did not block or delay muscle degradation at $8 \mathrm{~h} \mathrm{APF}^{28}$. We tested if a decrease in Tnmediated apoptosis could sensitize muscle cells to initiate autophagy as a compensatory mechanism to assure cell death. However, this does not seem to be the case as RNAi knockdown of Atg1, Atg5, or Atg18 does not further block DEOM histolysis in a tn RNAi background (Fig. S5). A second explanation is that the hypomorphic nature of these alleles may not completely abrogate Dark and Dronc function. Alternatively, additional effector caspases, including Dcp-1, Decay, and/or Damm, may be operating in the latter stages of DEOM histolysis since these caspases may function redundantly or act independent of the DIAP1-Drice axis ${ }^{3}$.

We expected more than a partial block in DEOM histolysis upon manipulation of DIAP1 (i.e., DIAP1 OE alone or tn RNAi+DIAP1 OE) at $12 \mathrm{~h} \mathrm{APF.} \mathrm{It} \mathrm{is} \mathrm{possible} \mathrm{that}$ normal or overexpressed DIAP1 levels in the DEOMs are not high enough to block apoptosis, especially using RNAi approaches to reduce Tn levels. However, the use of $t n-$ null alleles clearly shows a complete block in muscle degradation and a corresponding inhibition of active Dronc. Seemingly a delicate balance exists to regulate mRNA and protein expression, as well as protein turnover and proteolytic processing of active caspases. Cells must normally prevent cell death and only activate the apoptotic cascade upon a commitment to die. Thus, threshold levels of caspase activity must be reached for this terminal fate. There is evidence for stage or tissue-specific differential sensitivity to pro-apoptotic factors. Early L3 individuals are resistant to apoptosis, while wandering L3 larvae have elevated levels of Dark, Dronc, and Drice that are sufficient to trigger cell death under the appropriate stimuli $^{28}$. We propose a model whereby $\mathrm{Tn}$, through its RING domain, normally ubiquitinates DIAP1 for delivery to the proteasome during DEOM histolysis. This degradation of DIAP1 relieves Dronc inhibition, thereby initiating the caspase cascade for the execution of cell death (Fig. 8a). A general reduction in Tn, or loss of RING domain activity, prevents the addition of ubiquitin moieties and causes an increase in DIAP1 levels, effectively blocking cell death by limiting caspase activity (Fig. 8b).

Numerous roles have been identified for mammalian TRIM32 in normal and cancerous cells. In muscle, mutations in the NHL repeats result in limb-girdle muscular dystrophy type $2 \mathrm{H}$ or sarcotubular myopathy ${ }^{60-66}$. Several structural muscle proteins are targets of TRIM32 activity, including tropomyosin, desmin, $\alpha$-actinin, and dysbindin ${ }^{67-69}$. However, it is not yet clear if regulation of these muscle substrates contributes to normal muscle physiology, is required to prevent atrophy, or plays a critical role in disease pathology ${ }^{46}$. The TRIM32mediated degradation of additional protein substrates, including p53, Abi2, Piasy, and the X-linked IAP (XIAP), contribute to oncogenic or tumor suppressor activities that either confer resistance or susceptibility to apoptosis $^{70-73}$. Tumor necrosis factor- $\alpha$ can trigger death receptor-mediated apoptosis through the regulation of XIAP activity ${ }^{73}$. TRIM32 colocalizes and directly interacts with XIAP in human kidney epithelial cells (HEK293). Moreover, TRIM32 induces apoptosis through the direct ubiquitination and subsequent protein turnover of XIAP degradation ${ }^{73}$. This control of apoptotic cell death mirrors our genetic results, strongly suggesting that this TRIM32mediated regulation of IAP family members may be a conserved mechanism to regulate apoptosis. It would be interesting to further investigate if $\mathrm{Tn}$ and mammalian TRIM32 regulates apoptotic decisions in other contexts of muscle development and/or disease.

Herein, we have provided the first evidence for Tn in the regulation of muscle histolysis. Importantly, our genetic assays suggest that DIAP1 is a target of Tn and that regulation of DIAP1 and/or Caspase activity are crucial for a cell's decision to execute cell death. These 
findings will further increase our general understanding about PCD during tissue destruction in Drosophila development and will provide a conserved framework to identify novel targets of Tn.

\section{Materials and methods Fly genetics}

Drosophila melanogaster stocks were raised on standard cornmeal medium at $25^{\circ} \mathrm{C}$, unless otherwise indicated. The following fly stocks were used in this study: $w^{1118}$ strain as WT; mef2-Gal4 (Bloomington Drosophila Stock Center (BDSC), BL27390); $t n^{\Delta A 48}$, two different UAS- $t n$ RNAi lines (Vienna Drosophila Resource Center (VDRC), v19290 and v19291); UAS-EcR.B1 (BL6469); UAS-DIAP1. $H$ (BL6657); UAS-Dronc RNAi (BL32963) ${ }^{74}$; UAS-Dark RNAi (BL 33924); UAS-p35.BH2 (BL5073); UAS-tn FL ${ }^{48}$. The Dark $^{L 46}$ and Dronc $^{I 29}$ alleles were generously provided by Bergmann and co-workers ${ }^{53}$, 54 . The mef2-Gal4; UAS-tn RNAi (mef2>tn RNAi) line was created using standard recombination techniques and is maintained at $18{ }^{\circ} \mathrm{C}$ to maintain viability as $25^{\circ} \mathrm{C}$ results in partial pupal lethality.

\section{Immunostaining and microscopy}

To examine the effect of Tn in DEOM histolysis, white prepupae at $0 \mathrm{~h}$ were collected and aged until $8 \mathrm{~h}$ APF, $12 \mathrm{~h}$ APF, or 24 APF. Muscle preparations were dissected, fixed with $4 \%$ formaldehyde in phosphatebuffered saline (PBS) for $30 \mathrm{~min}$ and immunostained as indicated ${ }^{75}$. To analyze Tn function in salivary gland and midgut histolysis, wandering L3 larvae were dissected, fixed with $4 \%$ formaldehyde in PBS, and stained with DAPI (4',6-diamidino-2-phenylindole) and/or phalloidin. The following primary antibodies were used: rabbit anti-Caspase-3 (1:100, Cell Signaling Technology, Danvers, MA,USA), mouse anti-DIAP1 (1:200, B. $\mathrm{Hay}^{76}{ }^{7}$, guinea pig anti-p35 $(1: 10, \mathrm{P} . \mathrm{Meier})^{40}$, and guinea pig anti- $\mathrm{Tn}^{48}$. Secondary antibodies used for fluorescent immunolabeling were Alexa Fluor anti-mouse 488, Alexa Fluor anti-rabbit 488, Alexa Fluor antimouse 594, and Alexa Fluor anti-guinea pig 488 (1:400, Molecular Probes, Eugene, OR, USA). Phalloidin 488 and 594 were used for F-actin labeling (1:400, Molecular Probes, Eugene, OR, USA). Immunostained preparations were imaged on a Zeiss LSM 700, processed using the Zeiss Zen software and assembled into figures in Photoshop Elements.

\section{Western blotting}

Five to ten pupae of the appropriate genotype were homogenized in $3 \times$ Laemmli buffer $(150 \mathrm{mM}$ Tris- $\mathrm{HCl}$ (pH 6.8), $300 \mathrm{mM}$ dithiothreitol, 6\% sodium dodecyl sulfate (SDS), $0.3 \%$ bromophenol blue, and $30 \%$ glycerol), boiled at $100{ }^{\circ} \mathrm{C}$ for $10 \mathrm{~min}$, and centrifuged at $13,000 \times g$ to remove cellular debris. The resulting proteins were separated by SDS-polyacrylamide gel electrophoresis (SDS-PAGE), transferred to polyvinyl difluoride membrane, and probed with mouse anti-DIAP1 (1:500, B. Hays) or guinea pig anti-Dronc (1:400, P. Meier). Horse radish peroxidase-conjugated secondary antibodies (1:5000, GE Healthcare, UK) were used to detect the primary antibodies. Protein bands were visualized using the ECL Plus Western Blotting Detection Kit (ThermoFisher, Waltham, MA, USA) and analyzed with the FluorChem $M$ system (Protein Simple). The blots were stripped $(6.25 \mathrm{ml}$ of $1 \mathrm{M}$ Tris- $\mathrm{HCl}, \mathrm{pH} 6.8,10 \mathrm{ml}$ of $20 \%$ SDS, and $700 \mu \mathrm{l} \beta$-mercaptoethanol) and reprobed with mouse anti-ATP5 $\alpha$ (1:10,000, Abcam, UK). Densitometry analysis was performed by calculating the band intensities of DIAP1 relative to the ATP5 $\alpha$ loading control using Image J.

\section{Quantitative PCR}

RNA was isolated from a pool of five pupae using the RNAeasy Mini Kit (Qiagen, Valencia, CA, USA) for each genotype. Three separate pools were used for each time point. Either mef2-Gal4/+ (Fig. 2) or $w^{1118}$ (Fig. 6) were used as a control. After elution, RNA concentrations were determined and single strand complementary DNA (cDNA) was generated from $100 \mathrm{ng}$ of RNA using the SuperScript VILO cDNA Synthesis Kit (Invitrogen, Carlsbad, CA, USA). For the qPCR reactions, each cDNA sample was diluted to 1:50 and mixed with Power UP SYBR Green Master mix and the appropriate primers (Applied Biosystems, Foster City, CA, USA). rp49 was used as the reference gene. Primers were synthesized by Integrated DNA Technologies (IDT): rp49: 5'F-GCC CAAGGGTATCGACAACA-3'， 5R'-GCGCTTGTTCG ATCCGTAAC-3'; Dark: 5'F-AAGTACAATGTGAGC CGCCT-3', 5'R-CCCAAGTCTTTCCCGATCCC-3'; Dronc: 5'F-AGTCGGCCGATATTGTGGAC-3', 5'R-AC ATAAGGGGTGAGTGCTCC-3'; Drice: 5'F-GACTGC CGCTACAAGGACAT-3', 5'R-TGATTGGCCGTGAAGAAGCT-3'; Diap1: 5'F-GCGTGGAAATCGGTTGC TG-3', 5'R-GATGCGATCTAATGCTTCGGC-3'; $t n: 5^{\prime}$ F-GAGCTGCATATCGAAATCACCG-3'; ${ }^{\prime}$ R-AGATAG GCTTTTTCCGAGCAAAC-3'.

Three independent biological replicates were processed for each genotype and reactions were run in triplicate using the Quant Studio 3 Applied Biosystem with Quant studio design and analysis software. The average of the triplicates was used to calculate the $2^{-\Delta \Delta \mathrm{Ct}}$ values (normalized fold expression). Quantification of mRNA levels between different genotypes at the same time point was performed using multiple $t$ tests. Two-way analysis of variance (ANOVA) was used to compare transcript levels of the same genotype at different developmental time points. 


\section{Transgenic fly lines}

PCR amplification from the $t n$ cDNA GH06739 (Drosophila Genomic Resource Center) was used to generate

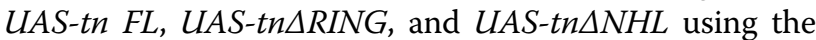
following primers: UAS-Tn FL-5'F-TAACGCGTCGACAATGGAGCAATTCGAGCAGCTGTTGAC-3', 5'R-C TAGTCTAGATCAGAAGACTTGGACGCGGTGATTC 3'; UAS-Tn $\triangle$ RING-5'F-AATAAGAATAGCGGCCGC ATGAATCTGCGACGAGACATCACG-3', 5'R- CTAG TCTAGATCAGAAGACTTGGACGCGGTGATTC 3'; UAS-Tn $\Delta$ NHL- $5^{\prime}$ F-TAACGCGTCGACAATGGAGCA ATTCGAGCAGCTGTTGAC 3'. 5'R-CTAGTCTACA TGCTGGCGCTTGCGCAGGTACACCTG $3^{\prime}$.

Each of the amplified regions was digested with SalI/ $X b a \mathrm{I}$ or $N o t \mathrm{I} / X b a \mathrm{I}$ (restriction sites underlined), subcloned into the pUAST Drosophila transformation vector, and verified by sequencing. Transgenic fly lines were generated by Genetic Services, Inc.

\section{Quantitative image analysis}

Initiation of muscle histolysis (\%): The number of DEOM1 muscles that were morphologically smaller or fragmented (indicative of histolysis initiation) in abdominal segments A2 and A3 in control or experimental genotypes at 0 or $8 \mathrm{~h}$ APF was quantitated. The percent of muscle histolysis initiation is illustrated as a percent of smaller or fragmented DEOM1 muscles/total number of DEOM1 muscles analyzed.

Histolysis (\%): The extent of muscle histolysis was determined by counting the number of DEOM1 muscles present in abdominal segments A2 and A3 in control and experimental samples at the indicated time points (12 or $24 \mathrm{~h} \mathrm{APF}$ ). All values are portrayed in percentages as DEOM1 muscles absent/total number of DEOM1 muscles analyzed.

Corrected total fluorescence (CTF): To measure the fluorescence intensities of Caspase-3, DIAP1, and p35 protein levels in dying DEOM1 or DEOM2 muscles, the CTF method was used ${ }^{77,78}$. The net average fluorescence intensity in a region of interest was measured in single section planes $(1 \mu \mathrm{m}$ slices $)$ inside the DEOMs and in an area without fluorescence for background subtraction. All measurements were performed in Image J.

Statistical analysis: All raw data were imported into GraphPad Prism 6.0 for statistical analysis and graph production. All error bars represent mean \pm standard error of the mean (SEM). Statistical significances were determined using either a Student's $t$ test, Mann-Whitney tests, or one-way ANOVA. Differences were considered significant if $p<0.05$ and are indicated in each figure legend. All " $n$ " values are listed in Supplementary Tables.

\begin{abstract}
Acknowledgements
We thank Bruce Hay and Pascal Meier for sharing antibodies. We also thank Andreas Bergmann, the VDRC, and the BDSC for fly lines used in this study. Monoclonal antibodies from the Developmental Studies Hybridoma Bank were created by the National Institute of Child Health and Human Development of the National Institutes of Health and are maintained at The University of lowa for monoclonal antibodies. This work was supported by a K-INBRE postdoctoral grant through the Institutional Development Award (IDeA) from the National Institute of General Medical Sciences (NIGMS) to K.V. (P20GM103418) and a grant through the National Institute of Arthritis and Musculoskeletal and Skin Diseases (NIAMS) to E.R.G (R01AR060788). Both are sponsored through the National Institutes of Health $(\mathrm{NIH})$.
\end{abstract}

\section{Conflict of interest}

The authors declare that they have no conflict of interest.

\section{Publisher's note}

Springer Nature remains neutral with regard to jurisdictional claims in published maps and institutional affiliations.

Supplementary Information accompanies this paper at (https://doi.org/ 10.1038/s41419-018-0756-x).

Received: 12 December 2017 Revised: 28 May 2018 Accepted: 30 May 2018 Published online: 03 July 2018

\section{References}

1. Fuchs, Y. \& Steller, H. Programmed cell death in animal development and disease. Cell 147, 742-758 (2011).

2. Fuchs, Y. \& Steller, H. Live to die another way: modes of programmed cell death and the signals emanating from dying cells. Nat. Rev. Mol. Cell. Biol. 16, 329-344 (2015).

3. Denton, D., Aung-Htut, M. T. \& Kumar, S. Developmentally programmed cell death in Drosophila. Biochim. Biophys. Acta 1833, 3499-3506 (2013).

4. Baehrecke, E. H. How death shapes life during development. Nat. Rev. Mol. Cell. Biol. 3, 779-787 (2002).

5. Jacobson, M. D., Weil, M. \& Raff, M. C. Programmed cell death in animal development. Cell 88, 347-354 (1997).

6. Kerr, J. F., Wyllie, A. H. \& Currie, A. R. Apoptosis: a basic biological phenomenon with wide-ranging implications in tissue kinetics. Br. J. Cancer 26, 239-257 (1972).

7. Yang, Z. \& Klionsky, D. J. An overview of the molecular mechanism of autophagy. Curr. Top. Microbiol. Immunol. 335, 1-32 (2009).

8. Walker, N. I., Harmon, B. V., Gobé, G. C. \& Kerr, J. F. Patterns of cell death. Methods Achiev. Exp. Pathol. 13, 18-54 (1988).

9. Nicolson, S., Denton, D. \& Kumar, S. Ecdysone-mediated programmed cell death in Drosophila. Int. J. Dev. Biol. 59, 23-32 (2015).

10. Baehrecke, E. H. Ecdysone signaling cascade and regulation of Drosophila metamorphosis. Arch. Insect Biochem. Physiol. 33, 231-244 (1996).

11. Baehrecke, E. H. Autophagic programmed cell death in Drosophila. Cell Death Differ. 10, 940-945 (2003).

12. Thummel, C. S. Flies on steroids-Drosophila metamorphosis and the mechanisms of steroid hormone action. Trends Genet. 12, 306-310 (1996).

13. Jiang, C., Baehrecke, E. H. \& Thummel, C. S. Steroid regulated programmed cell death during Drosophila metamorphosis. Development 124, 4673-4683 (1997).

14. Jiang, C., Lamblin, A. F., Steller, H. \& Thummel, C. S. A steroid-triggered transcriptional hierarchy controls salivary gland cell death during Drosophila metamorphosis. Mol. Cell 5, 445-455 (2000).

15. Lee, C. Y., Simon, C. R., Woodard, C. T. \& Baehrecke, E. H. Genetic mechanism for the stage- and tissue-specific regulation of steroid triggered programmed cell death in Drosophila. Dev. Biol. 252, 138-148 (2002).

16. DiBello, P. R., Withers, D. A., Bayer, C. A., Fristrom, J. W. \& Guild, G. M. The Drosophila broad-complex encodes a family of related proteins containing zinc fingers. Genetics 129, 385-397 (1991).

17. Cakouros, D., Daish, T., Martin, D., Baehrecke, E. H. \& Kumar, S. Ecdysoneinduced expression of the caspase DRONC during hormone-dependent programmed cell death in Drosophila is regulated by Broad-Complex. J. Cell Biol. 157, 985-995 (2002). 
18. Burtis, K. C., Thummel, C. S., Jones, C. W., Karim, F. D. \& Hogness, D. S. The Drosophila 74EF early puff contains E74, a complex ecdysone-inducible gene that encodes two ets-related proteins. Cell 61, 85-99 (1990).

19. Baehrecke, E. H. \& Thummel, C. S. The Drosophila E93 gene from the 93F early puff displays stage- and tissue-specific regulation by 20-hydroxyecdysone. Dev. Biol. 171, 85-97 (1995).

20. Daish, T. J., Cakouros, D. \& Kumar, S. Distinct promoter regions regulate spatial and temporal expression of the Drosophila caspase dronc. Cell Death Differ. 10, 1348-1356 (2003).

21. Kilpatrick, Z. E., Cakouros, D. \& Kumar, S. Ecdysone-mediated up-regulation of the effector caspase DRICE is required for hormone-dependent apoptosis in Drosophila cells. J. Biol. Chem. 280, 11981-11986 (2005).

22. Berry, D. L. \& Baehrecke, E. H. Growth arrest and autophagy are required for salivary gland cell degradation in Drosophila. Cell 131, 1137-1148 (2007).

23. Denton, D. et al. Autophagy, not apoptosis, is essential for midgut cell death in Drosophila. Curr. Biol. 19, 1741-1746 (2009).

24. Bate, M., Rushton, E. \& Currie, D. A. Cells with persistent twist expression are the embryonic precursors of adult muscles in Drosophila. Development 113, 79-89 (1991).

25. Currie, D. A. \& Bate, M. The development of adult abdominal muscles in Drosophila: myoblasts express twist and are associated with nerves. Development 113, 91-102 (1991).

26. Kimura, K. I. \& Truman, J. W. Postmetamorphic cell death in the nervous and muscular systems of Drosophila melanogaster. J. Neurosci. 10, 403-401 (1990).

27. Wasser, M., Bte Osman, Z. \& Chia, W. EAST and Chromator control the destruction and remodeling of muscles during Drosophila metamorphosis. Dev. Biol. 307, 380-393 (2007).

28. Zirin, J. et al. Ecdysone signaling at metamorphosis triggers apoptosis of Drosophila abdominal muscles. Dev. Biol. 383, 275-284 (2013).

29. Kuleesha, Y., Puah, W. C., Lin, F. \& Wasser, M. FMAj: a tool for high content analysis of muscle dynamics in Drosophila metamorphosis. BMC Bioinforma. 15(Suppl. 16), S6 (2014).

30. Kuleesha, Y., Puah, W. C. \& Wasser, M. Live imaging of muscle histolysis in Drosophila metamorphosis. BMC Dev. Biol. 16, 12 (2016).

31. Meier, P., Finch, A. \& Evan, G. Apoptosis in development. Nature 407, 796-801 (2000).

32. Thornberry, N. A. Caspases: key mediators of apoptosis. Chem. Biol. 5, R97-R103 (1998).

33. Mcllwain, D. R., Berger, T. \& Mak, T. W. Caspase functions in cell death and disease. Cold Spring Harb. Perspect. Biol. 5, a008656 (2013)

34. Salvesen, G. S. \& Duckett, C. S. IAP proteins: blocking the road to death's door Nat. Rev. Mol. Cell. Biol. 3, 401-410 (2002).

35. Kocab, A. J. \& Duckett, C. S. Inhibitor of apoptosis proteins as intracellular signaling intermediates. FEBS J. 283, 221-231 (2016).

36. Meier, P., Silke, J., Leevers, S. J. \& Evan, G. I. The Drosophila caspase DRONC is regulated by DIAP1. EMBO J. 19, 598-611 (2000).

37. Daish, T. J., Mills, K. \& Kumar, S. Drosophila caspase DRONC is required for specific developmental cell death pathways and stress-induced apoptosis. Dev. Cell 7, 909-915 (2004).

38. Waldhuber, M., Emoto, K. \& Petritsch, C. The Drosophila caspase DRONC is required for metamorphosis and cell death in response to irradiation and developmental signals. Mech. Dev. 122, 914-927 (2005)

39. Wang, S. L., Hawkins, C. J., Yoo, S. J., Müller, H. A. \& Hay, B. A. The Drosophila caspase inhibitor DIAP1 is essential for cell survival and is negatively regulated by HID. Cell 98, 453-463 (1999).

40. Yoo, S. J. et al. Hid, Rpr and Grim negatively regulate DIAP1 levels through distinct mechanisms. Nat. Cell Biol. 4, 416-424 (2002).

41. Goyal, L., McCall, K., Agapite, J., Hartwieg, E. \& Steller, H. Induction of apoptosis by Drosophila reaper, hid and grim through inhibition of IAP function. EMBO J. 19, 589-597 (2000).

42. Ryoo, H. D., Bergmann, A., Gonen, H., Ciechanover, A. \& Steller, H. Regulation of Drosophila IAP1 degradation and apoptosis by reaper and ubcD1. Nat. Cell Biol. 4, 432-438 (2002).

43. Hays, R., Wickline, L. \& Cagan, R. Morgue mediates apoptosis in the Drosophila melanogaster retina by promoting degradation of DIAP1. Nat. Cell Biol. 4, 425-431 (2002)

44. Yu, X., Wang, L., Acehan, D., Wang, X. \& Akey, C. W. Three-dimensional structure of a double apoptosome formed by the Drosophila Apaf-1 related killer. J. Mol. Biol. 355, 577-589 (2006).

45. Yuan, S. et al. Structure of the Drosophila apoptosome at 6.9 å resolution. Structure 19, 128-140 (2011).
46. Lazzari, E. \& Meroni, G. TRIM32 ubiquitin E3 ligase, one enzyme for several pathologies: from muscular dystrophy to tumours. Int. J. Biochem. Cell Biol. 79 469-477 (2016).

47. Shieh, P. B., Kudryashova, E. \& Spencer, M. J. Limb-girdle muscular dystrophy $2 \mathrm{H}$ and the role of TRIM32. Handb. Clin. Neurol. 101, 125-133 (2011).

48. LaBeau-DiMenna, E. M. et al. Thin, a Trim32 ortholog, is essential for myofibril stability and is required for the integrity of the costamere in Drosophila. Proc. Natl Acad. Sci. USA 109, 17983-17988 (2012).

49. Brooks, D. S., Vishal, K., Kawakami, J., Bouyain, S. \& Geisbrecht, E. R. Optimization of wrMTrck to monitor Drosophila larval locomotor activity. J. Insect Physiol. 9394, 11-17 (2016).

50. Yin, V. P. \& Thummel, C. S. Mechanisms of steroid-triggered programmed cell death in Drosophila. Semin. Cell Dev. Biol. 16, 237-243 (2005).

51. Fan, Y. \& Bergmann, A. The cleaved-Caspase-3 antibody is a marker of Caspase-9-like DRONC activity in Drosophila. Cell Death Differ. 17, 534-539 (2010).

52. Huh, J. R., Guo, M. \& Hay, B. A. Compensatory proliferation induced by cell death in the Drosophila wing disc requires activity of the apical cell death caspase Dronc in a nonapoptotic role. Curr. Biol. 14, 1262-1266 (2004).

53. Srivastava, M. et al. ARK, the Apaf-1 related killer in Drosophila, requires diverse domains for its apoptotic activity. Cell Death Differ. 14, 92-102 (2007).

54. Xu, D., Li, Y., Arcaro, M., Lackey, M. \& Bergmann, A. The CARD-carrying caspase Dronc is essential for most, but not all, developmental cell death in Drosophila. Development 132, 2125-2134 (2005).

55. Tocchini, C. \& Ciosk, R. TRIM-NHL proteins in development and disease. Semin Cell Dev. Biol. 47-48, 52-59 (2015).

56. Muro, I., Hay, B. A. \& Clem, R. J. The Drosophila DIAP1 protein is required to prevent accumulation of a continuously generated, processed form of the apical caspase DRONC. J. Biol. Chem. 277, 49644-49650 (2002).

57. Muro, I., Monser, K. \& Clem, R. J. Mechanism of Dronc activation in Drosophila cells. J. Cell Sci. 117, 5035-5041 (2004).

58. Lee, T. V. et al. Drosophila IAP1-mediated ubiquitylation controls activation of the initiator caspase DRONC independent of protein degradation. PLoS Genet. 7, e1002261 (2011).

59. Conradt, B. Genetic control of programmed cell death during animal development. Annu. Rev. Genet. 43, 493-523 (2009).

60. Frosk, P., Del Bigio, M. R., Wrogemann, K. \& Greenberg, C. R. Hutterite brothers both affected with two forms of limb girdle muscular dystrophy: LGMD2H and LGMD2I. Eur. J. Hum. Genet. 13, 978-982 (2005).

61. Schoser, B. G. et al. Commonality of TRIM32 mutation in causing sarcotubular myopathy and LGMD2H. Ann. Neurol. 57, 591-595 (2005).

62. Borg, K. et al. Intragenic deletion of TRIM32 in compound heterozygotes with sarcotubular myopathy/LGMD2H. Hum. Mutat. 30, E831-E844 (2009).

63. Cossée, M. et al. Use of SNP array analysis to identify a novel TRIM32 mutation in limb-girdle muscular dystrophy type 2H. Neuromuscul. Disord. 19, 255-260 (2009).

64. Nectoux, J. et al. Detection of TRIM32 deletions in LGMD patients analyzed by a combined strategy of $\mathrm{CGH}$ array and massively parallel sequencing. Eur. J. Hum. Genet. 23, 929-934 (2015).

65. Neri, M. et al. A patient with limb girdle muscular dystrophy carries a TRIM32 deletion, detected by a novel CGH array, in compound heterozygosis with a nonsense mutation. Neuromuscul. Disord. 23, 478-482 (2013).

66. Saccone, $\mathrm{V}$. et al. Mutations that impair interaction properties of TRIM32 associated with limb-girdle muscular dystrophy $2 \mathrm{H}$. Hum. Mutat. 29, 240-247 (2008).

67. Cohen, S., Zhai, B., Gygi, S. P. \& Goldberg, A. L. Ubiquitylation by Trim32 causes coupled loss of desmin, Z-bands, and thin filaments in muscle atrophy. J. Cell Biol. 198, 575-589 (2012).

68. Kudryashova, E., Kudryashov, D., Kramerova, I. \& Spencer, M. J. Trim32 is a ubiquitin ligase mutated in limb girdle muscular dystrophy type $2 \mathrm{H}$ that binds to skeletal muscle myosin and ubiquitinates actin. J. Mol. Biol. 354, 413-424 (2005).

69. Locke, M., Tinsley, C. L., Benson, M. A. \& Blake, D. J. TRIM32 is an E3 ubiquitin ligase for dysbindin. Hum. Mol. Genet. 18, 2344-2358 (2009).

70. Kudryashova, E., Kramerova, I. \& Spencer, M. J. Satellite cell senescence underlies myopathy in a mouse model of limb-girdle muscular dystrophy $2 \mathrm{H}$. J. Clin. Invest. 122, 1764-1776 (2012).

71. Liu, J. et al. E3 ubiquitin ligase TRIM32 negatively regulates tumor suppressor p53 to promote tumorigenesis. Cell Death Differ. 21, 1792-1804 (2014).

72. Lin, T. Y., Huang, C. H., Chou, W. G. \& Juang, J. L. Abi enhances Abl-mediated CDC2 phosphorylation and inactivation. J. Biomed. Sci. 11, 902-910 (2004). 
73. Ryu, Y. S. et al. TRIM32 protein sensitizes cells to tumor necrosis factor (TNFa)induced apoptosis via its RING domain-dependent E3 ligase activity against Xlinked inhibitor of apoptosis (XIAP). J. Biol. Chem. 286, 25729-25738 (2011).

74. Qi, Y., Liu, H., Daniels, M. P., Zhang, G. \& Xu, H. Loss of Drosophila i-AAA protease, dYME1L, causes abnormal mitochondria and apoptotic degeneration. Cell Death Differ. 23, 291-302 (2016).

75. Zirin, J., Nieuwenhuis, J. \& Perrimon, N. Role of autophagy in glycogen breakdown and its relevance to chloroquine myopathy. PLoS Biol. 11 e1001708 (2013)
76. Wilson, R. et al. The DIAP1 RING finger mediates ubiquitination of Dronc and is indispensable for regulating apoptosis. Nat. Cell Biol. 4, 445-450 (2002).

77. Burgess, A. et al. Loss of human Greatwall results in $G 2$ arrest and multiple mitotic defects due to deregulation of the cyclin B-Cdc2/PP2A balance. Proc. Natl Acad. Sci. USA 107, 12564-12569 (2010).

78. McCloy, R. A. et al. Partial inhibition of Cdk1 in G2 phase overrides the SAC and decouples mitotic events. Cell Cycle 13, 1400-1412 (2014). 